\title{
STRUCTURE AND FUNCTION OF THE FRUIT MICROBIOME IN HEALTHY AND DISEASED KIWIFRUIT
}

\author{
Wenneng Wu ${ }^{1, \#, ~ J i q i n g ~ L e i ~}{ }^{1, \#, ~ M u z a m m i l ~ H u s s a i n ~}{ }^{2}$, Sen $\mathrm{Cao}^{1}$, Bin Du${ }^{1}$ and Rui Wang, ${ }^{1, *}$ \\ ${ }^{1}$ Food and Pharmaceutical Engineering Institute, Guiyang College, Guiyang 550003, People's Republic of China; \\ ${ }^{2}$ State Key Laboratory of Mycology, Institute of Microbiology, Chinese Academy of Sciences, No 3 Park 1, Beichen \\ West Rd., Chaoyang District, Beijing 100101, China. \\ \#These authors contributed equally to this work. \\ "Corresponding author's e-mail: wr12581@126.com
}

\begin{abstract}
The fruit surface is an infection court where foodborne pathogens compete with indigenous microbiota for microsites to invade the fruits for nutrients acquisition. However, our current understanding of the structure and functions of fruit microbiome vis$a$-vis postharvest pathogen infection is still nascent. Here, we sequenced the metagenomic DNA to understand the structural and functional attributes of healthy and diseased kiwifruit microbiome. The healthy fruits exhibited higher microbial diversity and distinct microbiome composition compared with diseased fruits. The microbiome of diseased fruit was dominated by fungal pathogens Neofusicoccum parvum and Diplodiaseriata, while the microbiome of healthy fruits were enriched by bacteria from Methylobacteriaceae, Sphingomonadaceae, Nocardioidaceae and fungi in Pleosporaceae. Importantly, the healthy fruit microbiome had a higher relative abundance of genes related to $\mathrm{ABC}$ transporter, two-component system, bacterial chemotaxis, bacterial secretion system, but had a lower relative abundance of genes associated with polycyclic aromatic hydrocarbon degradation, amino sugar and nucleotide sugar metabolism, glycine, serine and threonine metabolism compared with diseased fruits. Our results indicate that pathogen infection disrupts the fruit microbiome. The changes in microbiome composition and functions could also increase the possibility of secondary pathogen infection as the reduced microbial diversity may demonstrate less resistance to pathogens infection. Therefore, monitoring the microbiome dynamics and their functions using metagenomic approaches could be useful to build a predictive understanding of accurate postharvest disease diagnosis and management in the future.
\end{abstract}

Keywords: Kiwifruit, postharvest pathogen, fruit microbiome, shotgun metagenomic sequencing, microbial communities, functional potential

\section{INTRODUCTION}

Soil microorganisms preferentially colonize different parts of plant and play pivotal role in plant nutrition (Bulgarelli et al., 2013), disease protection (Santhanam et al., 2015; Ritpitakphong et al., 2016; Hussain et al., 2018) and overall vigor (Berendsen et al., 2012; Panke-Buisse et al., 2015). Recently, high-throughput sequencing approaches have provided deep insights into structure and functions of rootassociated and leaf microbiota (Bulgarelli et al., 2015; Bai et al., 2015), but our current understanding about the fruit microbiome is still evolving. Fruits are believed to harbor diverse microorganisms as epiphytes on the surface and endophytes within tissues (Preto et al., 2017; Droby and Wisniewski, 2018), thereby are susceptible to infection by various pathogens before and after harvest (Hamid et al., 2014; Zhou et al., 2015; Hussain et al., 2016; Hayat et al., 2017). A detailed investigation of grapevine-associated microbiota revealed that leaves, flowers and fruit bacterial communities share a higher proportion of microbial taxa with the soil communities, pointing out that soil not only serves as a start inoculum for the establishment of plant-associated belowground bacterial communities but also for aboveground communities (Zarraonaindia et al., 2015). Several studies demonstrated that the fruit surface host several microbes that restrict foodborne pathogens infection (Preto et al., 2017; Drobyet al., 2009; Habiba et al., 2019), similar to plant roots and leaves which host microbes for disease protection (Ritpitakphong et al., 2016; Mendes et al., 2011; Hamid et al., 2017; Mwaheb et al., 2017). This understanding paved the way for the development of several commercial biocontrol products (Droby and Wisniewski, 2018).

Recent studies employing next-generation sequencing approaches provided new insights into the microbial ecology of fruits including, grapes (Zarraonaindia et al., 2015; Chou et al., 2018), apple (Abdelfattah et al., 2016), mango (Diskin et al., 2017) and olive (Abdelfattah et al., 2018). Detailed investigation of microbial communities revealed that the fruits microbiota are taxonomically structured and characterized by the members of main bacterial phyla Proteobacteria, Bacteroidetes and Firmicutes (Zarraonaindia et al., 2015), and fungal phyla Ascomycota and 
Basidiomycota (Abdelfattah et al., 2018). It has been observed that the under-vine management alters the structure of soil bacterial and fungal microbiota but did not induce changes in the composition of the grape-associated microbiota (Chou et al., 2018). However, it is interesting to note that the fruit parts such as peel, wound, stem-end, and calyx-end of apple have been found to vary in fungal microbiota composition (Abdelfattah et al., 2016). A recent study on mango showed that the microbial community composition of fruit-associated microbiota changed appreciably with temperature and storage duration (Diskin et al., 2017). Moreover, the increased abundance of stem-end rot pathogens was closely correlated with the increased abundance of chitin-degrading bacterial family Chitinophagaceae (Diskin et al., 2017). These studies have given a new direction to further understand the fruit microbiome structure and those factors which shape them. However, the research on fruit microbiome is still at initial stages and key questions regarding how postharvest pathogens alter the structure and functions of fruit microbiome are yet to be answered.

Kiwifruit, a member of the family Actinidiaceae is a widely grown horticultural crop worldwide (Zhou et al., 2015; Polat et al., 2017), and its fruits are a rich source of vitamins, carotenoids and phenolics (Latocha et al., 2013). However, postharvest pathogens are considered to cause significant losses in kiwifruit (Mari et al., 2015). Hitherto, several microbial antagonists have been demonstrated to suppress fruit rots of kiwifruit (Di Francesco et al., 2018; Tang et al., 2015; Cook et al., 1999). Although fruit-associated microbes have been an area of many investigations for decades (Droby et al., 2009), still no clear information on the exhaustive characterization of fruit microbiome and their functional attributes is available. Here, we sequenced metagenomic DNA of the fruit microbiome from healthy and diseased kiwifruit to address two basic questions: Does the microbiota composition of healthy fruits differ compared with diseased fruits? What functional attributes differentiate healthy and diseased fruits microbiota?

\section{MATERIALS AND METHODS}

Collection of healthy and diseased fruits: Kiwifruits (Actinidia deliciosa) were harvested from the trees grown in fields located at Xiuwen County, Guiyang city, Guizhou province of China. Fruits with uniform size and without blemish were separated, washed with tap-water and air-dried. Next, the cardboard boxes were used to place fruits and were transferred to shelf-life storage temperature at $0 \pm 0.5^{\circ} \mathrm{C}$. After 60 days, the healthy fruits without blemishes and the fruit that were completely rotten displayed diseased symptoms were separated and placed in coolbox for tissue sampling.

Tissue sampling and DNA extraction: The fresh tissues from healthy fruits and decayed tissues from diseased kiwifruits were sampled using a sterile surgical blade. A $10 \mathrm{~mm}^{2}$ fruit tissue from 4 sides of fruit $(10 \mathrm{~mm}$ external and $10 \mathrm{~mm}$ internal) were sampled and combined for each fruit sample. A total of 3 samples for healthy and diseased kiwifruit were prepared. All healthy and diseased fruits samples were then ground with mortar and pestle in liquid nitrogen and stored at $-80^{\circ} \mathrm{C}$ until DNA extraction. The FastDNA Spin Kit for Soil (MP Biomedicals) was used for the extraction of DNA by following the manufacturer's instructions. Fruit samples (1 g) were homogenized in the FastPrep instrument for $40 \mathrm{~s}$ at a speed setting of 6.0. The extracted DNA was eluted in $50 \mu \mathrm{L}$ of elution buffer and then stored at $-80^{\circ} \mathrm{C}$ for shotgun metagenomic sequencing.

\section{Library preparation and shotgun metagenomic sequencing:} Next-generation sequencing library preparations were constructed following the manufacturer's protocol (NEBNext Ultra II DNA Library Prep Kit for Illumina). For each sample, $200 \mathrm{ng}$ genomic DNA was randomly fragmented to $<500 \mathrm{bp}$ by sonication (Covaris S220). The fragments were treated with End Prep Enzyme Mix to repair both ends and add a dAtailing in one reaction, followed by a T-A ligation to add adaptors to both ends. Size selection of Adaptor-ligated DNA was then performed using AxyPrep Mag PCR Clean-up (Axygen), and fragments of $\sim 410 \mathrm{bp}$ (with the approximate insert size of $350 \mathrm{bp}$ ) were recovered. Each sample was then amplified by PCR for 8 cycles using P5 and P7 primers, with both primers carrying sequences which can anneal with flow cell to perform bridge PCR and P7 primer carrying a six-base index allowing for multiplexing. The PCR products were cleaned up using AxyPrep Mag PCR Cleanup (Axygen), validated using an Agilent 2100 Bioanalyzer (Agilent Technologies, Palo Alto, CA, USA), and quantified by Qubit 3.0 Fluorometer (Invitrogen, Carlsbad, CA, USA). Then libraries with different indexes were multiplexed and loaded on an Illumina HiSeq instrument according to manufacturer's instructions (Illumina, San Diego, CA, USA). Sequencing was carried out using a 2x150 paired-end (PE) configuration; image analysis and base calling were conducted by the HiSeq Control Software (HCS) + OLB + GAPipeline-1.6 (Illumina) on the HiSeq instrument.

Data analysis and statistics: Raw shotgun sequencing reads were trimmed using cutadapt. Low-quality reads, N-rich reads and adapter-polluted reads were removed. Then host contamination reads were removed using BWA. Samples were each assembled de novo to obtain separate assemblies. Whole genome de novo assemblies were performed using MEGAHIT with different k-mer. The best assembly result of Scaffold, which has the largest N50, was selected for the gene prediction analysis. Genes of each sample were predicted using Prodigal. CD-HIT was used to cluster genes derived from all samples with a default identity of 0.95 and coverage of 0.9. In order to analyze the relative abundance of unigenes in each sample, paired-end clean reads were mapped to unigenes using SOAPAligner to generate read coverage 
information for unigenes. Gene abundance was calculated based on the number of aligned reads and normalized to gene length. Scaftigs from each sample were used for open reading frame (ORF) prediction through MetaGeneMark. In order to explore the microbial composition of the samples, we constructed a sequence database of bacteria, fungi, archaea and viruses from the NT database of NCBI. The unigene sequences were blasted against the constructed microbial database. The lowest common ancestor (LCA) was determined using Metagenome Analyzer. The abundance of a species in one sample equals the sum of the gene abundance annotated for the species. Diamond (version v0.8.15.77) was used to search the protein sequences of the unigenes with the Kyoto Encyclopedia of Genes and Genomes (KEGG) database with $\mathrm{E}<1 \mathrm{e}-5$. The statistical significance threshold of the sequence alignment was set at $1 e-5$ and the sequence alignment length was set as no less than $60 \%$ of the reference gene protein length. The matched result with best scores was selected for annotation. To determine the similarity or difference of taxonomic and functional components between different samples, relative clustering analysis and principal component analysis (PCA) were performed.

\section{RESULTS AND DISCUSSION}

Sample description and general information of the datasets: Preliminary morphological examination showed that the stored disease kiwifruits were soft, watery and severely rotten indicating postharvest microbial infection. However, the healthy fruits were fresh and no rot symptoms were observed on the surface and interior of the fruit. To identify the kiwifruit postharvest pathogens and to compare the structure and function of microbiota, we sequenced metagenomic DNA of the healthy and diseased fruit microbiome using shotgun metagenomic approach. We retrieved a total of 189.85 million clean reads from all samples with an average of 31.64 million reads per sample and the ratio of clean reads to $\mathrm{PF}$ reads were $22.499 \%$. Sequence assembly with the SOAPdenovo software revealed 176291, 128880, 157096 total sequences for healthy fruits samples and 19615, 19229, 27095 total sequences for diseased fruit samples with the scaftigs longer than $200 \mathrm{bp}$, respectively. Furthermore, gene prediction using MetaGeneMark software showed that there was on average 155018 total sequences for healthy fruits samples and 49650 total sequences for diseased fruit samples with N50 lengths were $258,267,270$ for healthy fruits and $657,723,663$ for diseased fruits.

Diversity and structure of the healthy and diseased fruit microbiome: The alpha-diversity (within-sample diversity) of the fruit microbiome in healthy and diseased kiwifruits was estimated using Simpson's diversity index. The diversity values of the bacterial and fungal microbiota were significantly higher in the healthy fruits than those in the fruits that had been infected by foodborne pathogen (Fig. 1). In previous studies, it has been documented that the infection of plant roots by pathogens significantly disrupt the microbiome diversity leading to predicted community functions (Hussain et al., 2018; Wei et al., 2018). This indicates that the pathogens infection does not only disrupt the belowground microbial communities but also the aboveground fruits inhabiting microbial communities. Furthermore, the drastic change in microbiome diversity was comparable to the shift in microbiome composition as evident from the principal component analysis (PCA), where the healthy and diseased fruit samples were clearly separated from each other (Fig. 2).

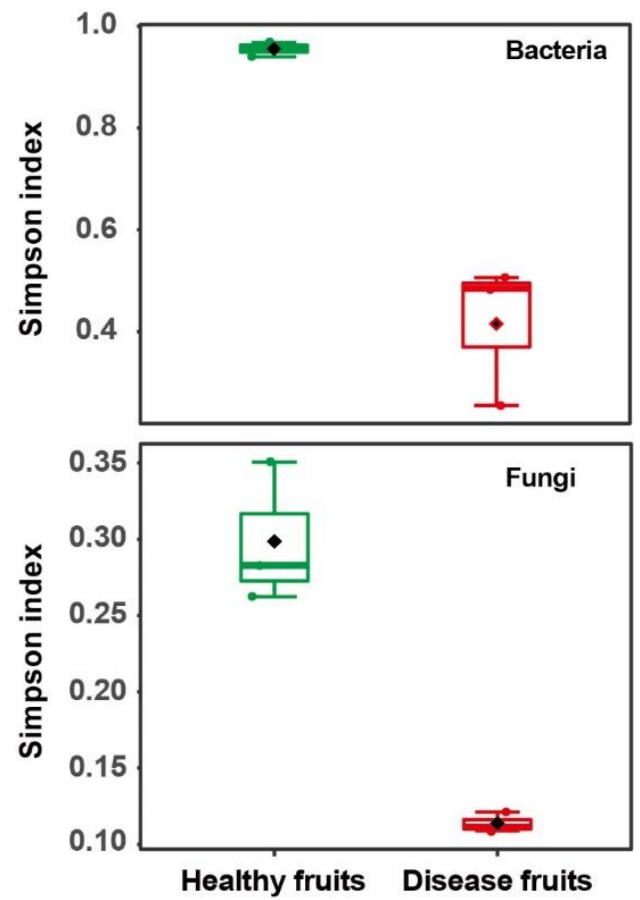

Figure 1.Diversity of the microbiome in the healthy and diseased kiwifruits. Alpha-diversity of the fungal and bacterial community in healthy and diseased fruits was estimated based on the Simpson index.
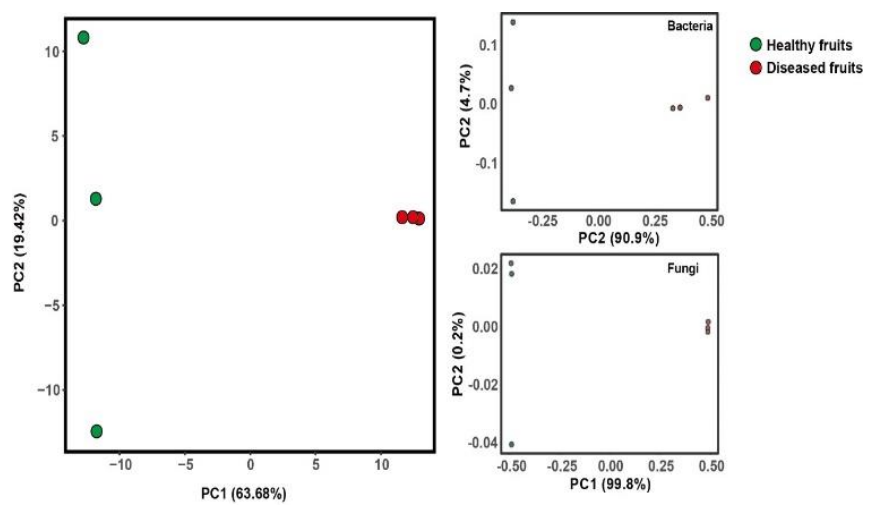

Figure 2. Fruit microbiome variation in healthy and diseased kiwifruits. 
Principal component analysis (PCA) of fruit microbiome revealed a clear separation between healthy and diseased fruit samples. The independent PCA analysis of bacterial and fungal communities showed a similar pattern of separation between healthy and diseased fruits.

Importantly, we observed notable differences in the composition of the key bacterial and fungal microbiota inhabiting healthy and diseased fruits. Taxonomic assignment at phylum level indicated that Proteobacteria, Actinobacteria and Bacteroidetes from the bacterial domain, and Ascomycota and Basidiomycota from the fungal domain largely dominated the fruit microbiome of kiwifruit (Fig. 3a).

a
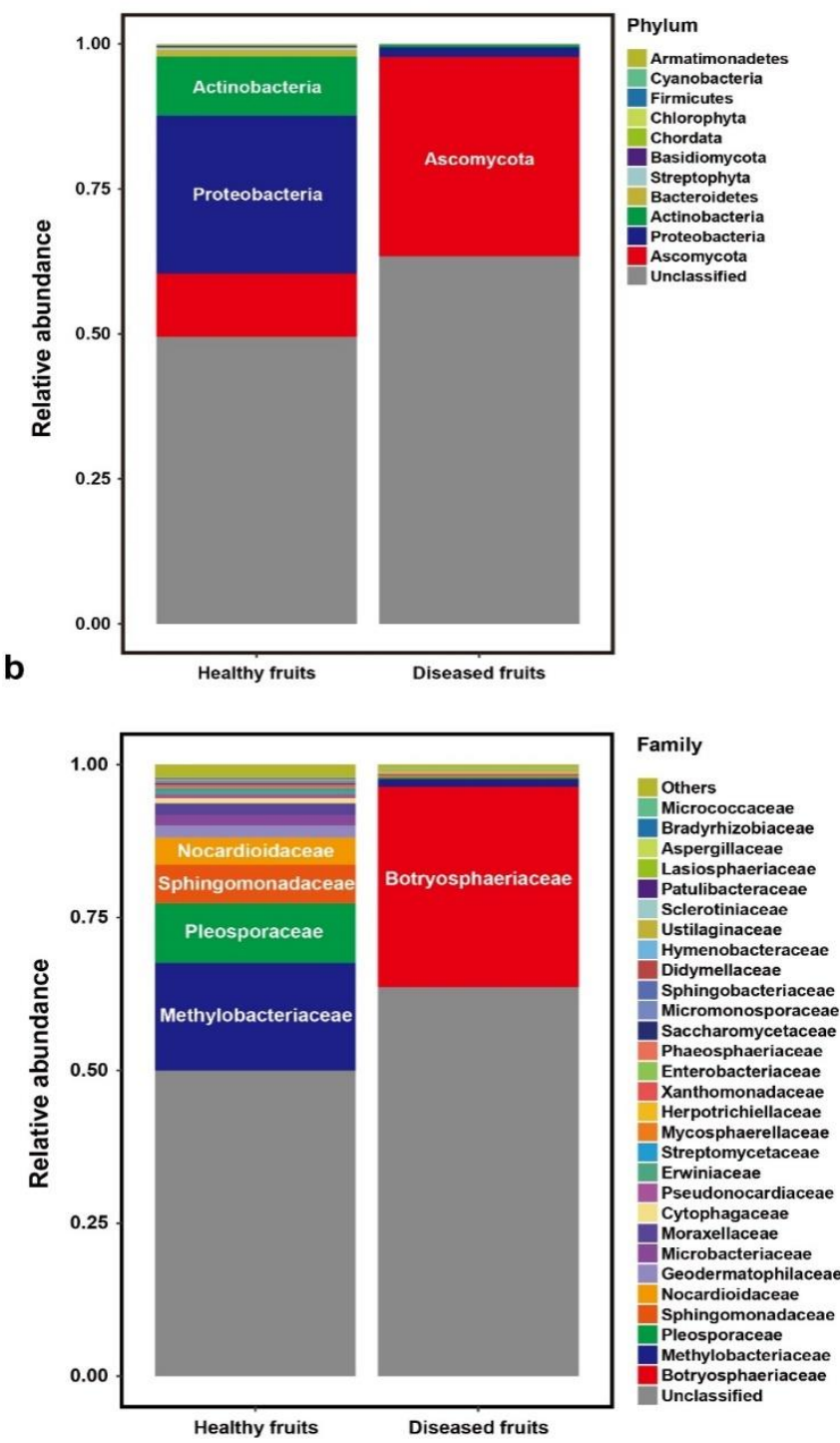

Figure 3. Microbiome composition in healthy and diseased fruits. (a) Relative abundance of bacterial and fungal phyla in healthy and diseased fruit samples. (b) Relative abundance of bacterial and fungal families in healthy and diseased fruits.
Consistent with the dominance of these phyla in the plant root microbiome (Bulgarelli et al., 2012; Urbina et al., 2018), the fruits microbiome can also be characterized by the cooccurrence of 3 bacterial phyla Proteobacteria, Actinobacteria and Bacteroidetes and 2 fungal phyla Ascomycota and Basidiomycota. It has been previously reported that fruitassociated microbiota share a higher proportion of microbial taxa with the soil communities, highlighting that soil not only serves as start inoculum for the establishment of plantassociated belowground bacterial communities but also for aboveground communities (Zarraonaindia et al., 2015). The density dynamic of these abundant phyla was significantly different between healthy and diseased fruits. At the phylum level, the healthy fruits were enriched by bacteria (Proteobacteria and Actinobacteria), while the diseased fruits were dominated by fungi (Ascomycota) (Fig. 3a). At the family level, bacteria from Methylobacteriaceae, Sphingomonadaceae, Nocardioidaceae, Geodermatophilaceae, Microbacteriaceae, Moraxellaceae, Cytophagaceae were more abundant in the healthy fruits than diseased fruits (Fig. 3b). In contrast, the fungal family Pleosporaceae was abundant in healthy fruits, while the Botryosphaeriaceae was dominant in diseased fruit microbiota (Fig. 3b). Members of the family Botryosphaeriaceae are well-recognized pathogens of fruit (Zhou et al., 2015).

In order to gain further insight into the composition of microbial taxa and foodborne pathogens, we compared the microbiota of healthy and diseased fruits at the genus and species level. We found that the fruit pathogenic fungal species Neofusicoccum parvum and Diplodia seriata from the family Botryosphaeriaceae had clearly much higher relative abundance in the diseased fruits than healthy fruits (Fig. 4a,b; Fig. 5).

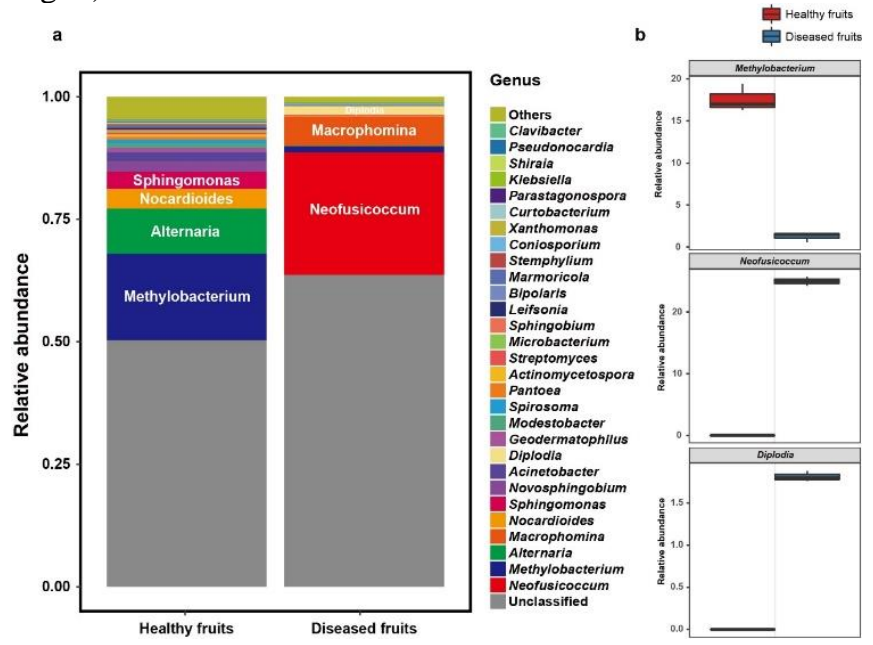

Figure 4. Microbial genera in healthy and diseased fruits. (a) Relative abundance of bacterial and fungal genera in healthy and diseased fruit. (b) Relative abundance of dominant key taxa in healthy and diseased fruits. 


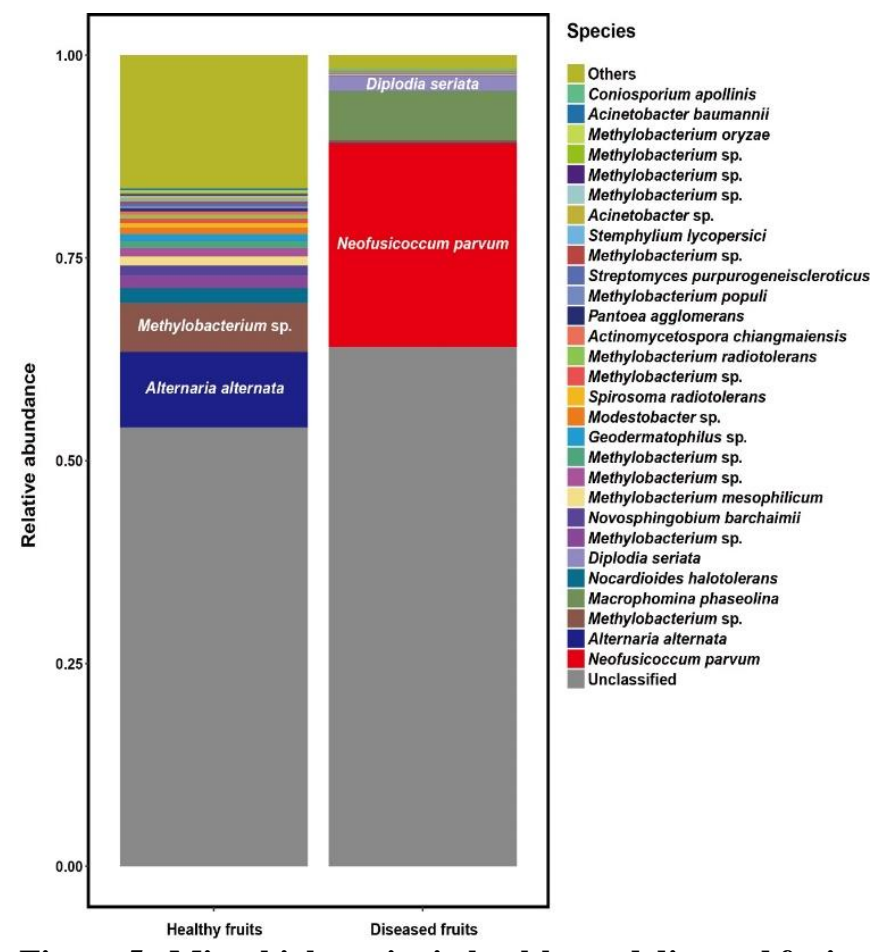

Figure 5. Microbial species in healthy and diseased fruits. (a) Relative abundance of bacterial and fungal species in healthy and diseased fruit.
It has been previously reported that the fungal species Neofusicoccum parvum is a known causative agent of postharvest disease in kiwifruit (Zhou et al., 2015). The fungus D. seriata infects fruit of apple (Kim et al., 2016), grape (Lorenzini and Zapparoli, 2018), loquat (Abbas and Naz, 2018) and olive (Moral et al., 2008), but D. seriatahas not been previously reported on kiwifruit, thereby demonstrating that metagenomic approaches provide deeper insight into the previously unrecognized pathogenic microbes of the fruits. In contrast, the healthy fruits harbor diverse bacterial genera inter alia, Methylobacterium, Nocardioides, Sphingomonas, Novosphingobium, Acinetobacter, Geodermatophilus, Modestobacter and Spirosoma (Fig. 4a). Taken together, these results indicate that the fruit microbiota diversity is severely hampered by foodborne pathogen infection in diseased fruit and leads to sweeping changes in microbial community composition.

Functional genes of the microbiome in healthy and diseased kiwifruit: The separation patterns of the microbial functional genes in the ordination plots for healthy and diseased fruits were nearly identical to the patterns determined for the taxonomic composition of the healthy and diseased fruits in the beta-diversity analysis (Fig. 6a). Despite these differences, several gene categories were unique to healthy as well as diseased fruits, as observed in the venn diagram (Fig. 6b), e.g. the healthy fruits had genes associated with

Table 1. KEGG ortholog (KO) groups exclusively detected from healthy and diseased kiwifruits, as shown in Figure 5b.

\begin{tabular}{|c|c|}
\hline Tealthy fruits & Diseased fruits \\
\hline ro00290__Valine,_leucine_and_isoleucine_biosynthesis & ko05144__Malaria \\
\hline ro05143_African_trypanosomiasis & ko04611_Platelet_activation \\
\hline o04080_Neuroactive_ligand-receptor_interaction & ko04970_Salivary_secretion \\
\hline co04390_Hippo_signaling_pathway & $\begin{array}{l}\text { ko00604_Glycosphingolipid_biosynthesis_- } \\
\text { ganglio_series }\end{array}$ \\
\hline co04115__p53_signaling_pathway & ko04724_Glutamatergic_synapse \\
\hline c001055_Biosynthesis_of_vancomycin_group_antibiotics & ko04512_ECM-receptor_interaction \\
\hline ro00121_Secondary_bile_acid_biosynthesis & $\begin{array}{l}\text { ko04961_Endocrine_and_other_factor } \\
\text { regulated_calcium_reabsorption }\end{array}$ \\
\hline co00472_D-Arginine_and_D-ornithine_metabolism & ko04911_Insulin_secretion \\
\hline co00363_Bisphenol_degradation & ko04140_Regulation_of_autophagy \\
\hline c000902_Monoterpenoid_biosynthesis & ko04722_Neurotrophin_signaling_pathway \\
\hline $\begin{array}{l}\text { co0532_Glycosaminoglycan_biosynthesis_- } \\
\text { chondroitin_sulfate_/_dermatan_sulfate }\end{array}$ & ko04360_Axon_guidance \\
\hline $\begin{array}{l}\text { c00534_Glycosaminoglycan_biosynthesis_- } \\
\text { heparan_sulfate_/_heparin }\end{array}$ & ko04630_Jak-STAT_signaling_pathway \\
\hline co00943_Isoflavonoid_biosynthesis & ko04964_Proximal_tubule_bicarbonate_reclamatior \\
\hline c005120_Epithelial_cell_signaling_in_Helicobacter_pylori_infection & ko04330_Notch_signaling_pathway \\
\hline c05152_Tuberculosis & ko05162_Measles \\
\hline c001502_Vancomycin_resistance & ko00512_Mucin_type_O-Glycan_biosynthesis \\
\hline \multirow[t]{4}{*}{ c003320_PPAR_signaling_pathway } & ko04114_Oocyte_meiosis \\
\hline & ko00403_Indole_diterpene_alkaloid_biosynthesis \\
\hline & ko04622_RIG-I-like_receptor_signaling_pathway \\
\hline & ko04726_Serotonergic_synapse \\
\hline
\end{tabular}


biosynthesis of amino acids, monoterpenoid, glycosaminoglycan and antibiotics, signaling pathways (p53, Hippo, PPAR), D-Arginine and D-ornithine metabolism and others compared with the diseased fruits which had genes associated with Jak-STAT signaling pathway, indolediterpene alkaloid biosynthesis, regulation of autophagy and others (Table 1; Fig. 6b).

a

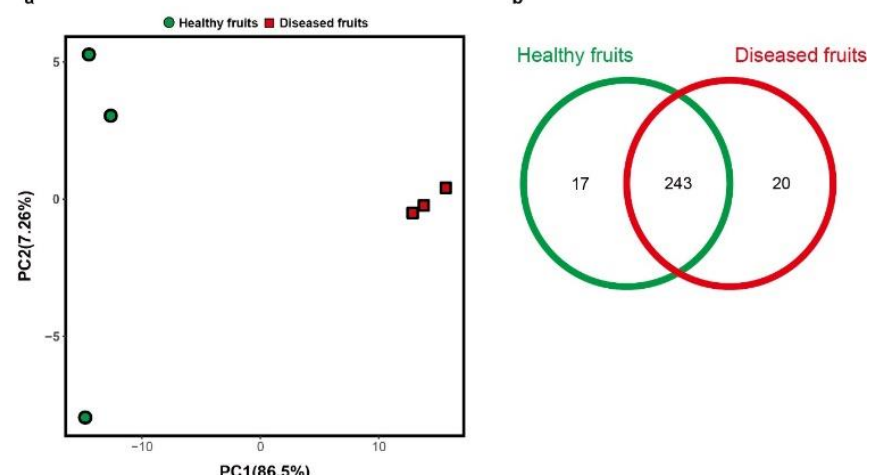

Figure 6. Variation in KEGG orthologs group for healthy and diseased fruits. (a) Principal component analysis (PCA) using the relative abundances of KEGG ortholog (KO) groups showed separation between healthy and diseased kiwifruit samples. (b) Venn diagram of functional gene categories showed unique as well as shared functional microbial genes in healthy and diseased fruit.

The greater differences between healthy and diseased fruits microbiota were also observed by comparing the relative abundances of functional gene categories. Mostly the gene categories abundant in the healthy fruits compared with diseased fruits were associated with metabolism functions (Fig. 7, 8).

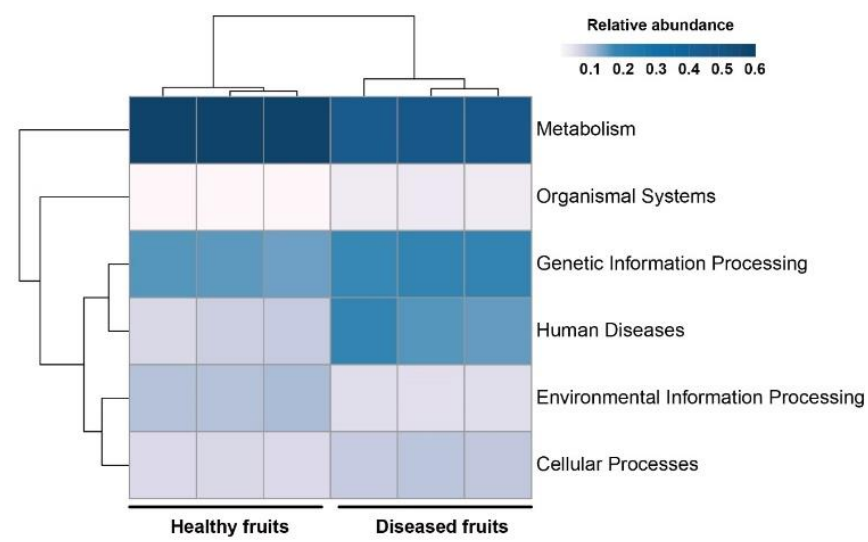

Figure 7. Heat map showing relative abundance of the level 1 KEGG ortholog (KO) groups associated with healthy and diseased fruits.

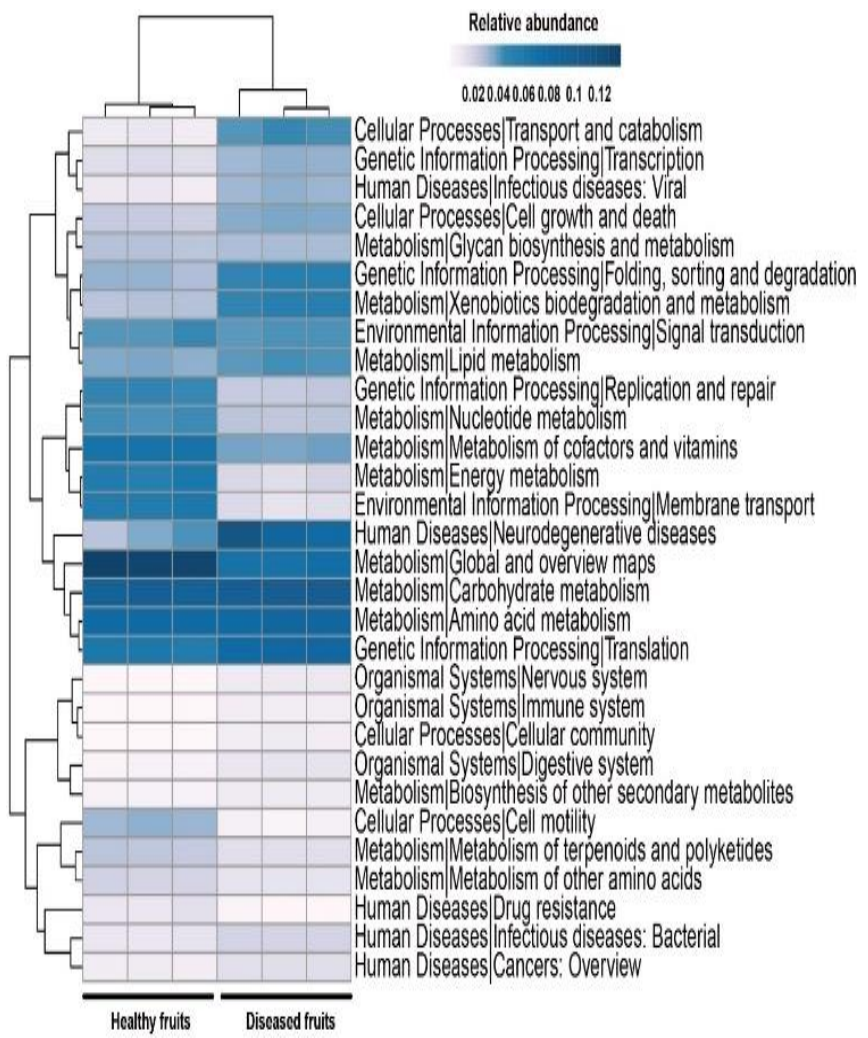

Figure 8. Heat map showing relative abundance of the level 2 KEGG ortholog groups associated with healthy and diseased fruits.

Compared with diseased fruits, genes associated with $\mathrm{ABC}$ transporters, a two-component system, sulfur metabolism, cell cycle, bacterial chemotaxis, oxidative phosphorylation, bacterial secretion system, homologous recombination and porphyrin and chlorophyll metabolism had more relative abundance in the healthy fruits (Fig. 9). The high relative abundance of ABC transporters genes (defense mechanism) in microbiome reflected an increased tolerance to antimicrobial compounds (Wu et al., 2018). Moreover, the increased abundance of genes related to two-component system contributes to biofilm formation in bacteria (Tiwari et $a l ., 2017)$, indicating that the biofilm formation by bacteria on fruit surface could reduce the availability of microsites and also elevate the competition for nutrients between microbes and postharvest pathogens. Interestingly, the higher abundance of bacterial chemotaxis genes reflects that the bacteria assembling in the environment had a lower concentration of toxic chemicals (Wadhams and Armitage, 2004), thereby confirming that the healthy fruits contain more concentration of beneficial chemicals contributing to higher microbial diversity. Also, we found an increased relative abundance of bacterial secretion system genes in healthy fruits. 


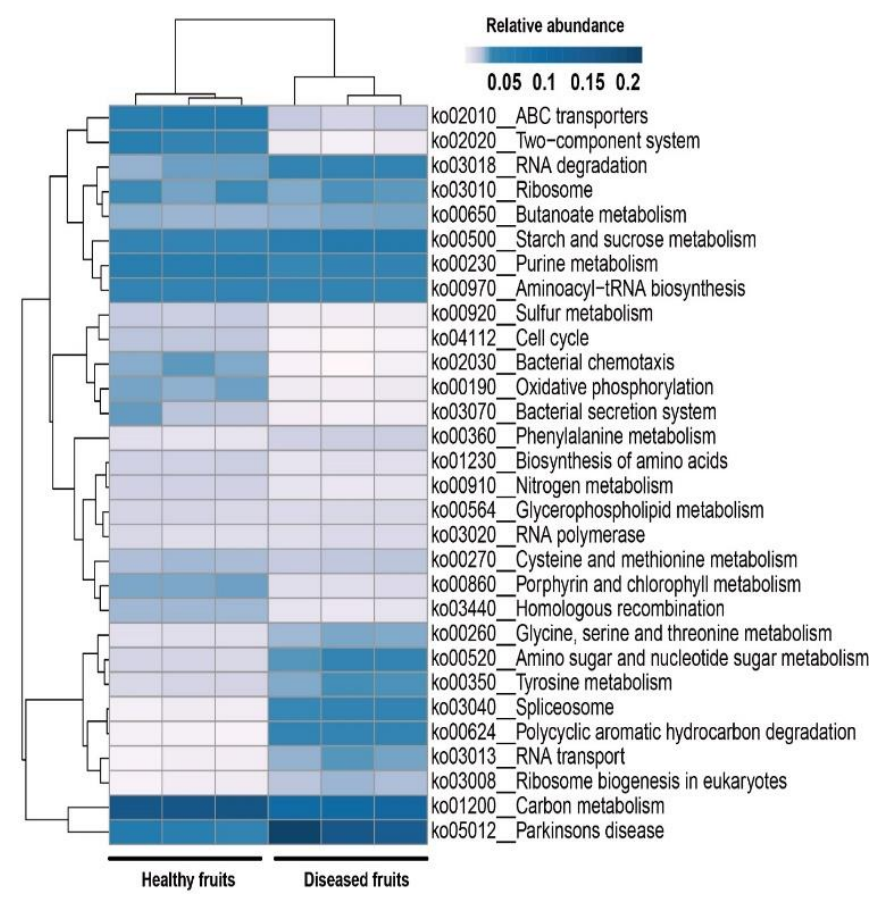

Figure 9. Heat map showing relative abundance of the top 30 most abundant level 3 KEGG ortholog groups associated with healthy and diseased fruits.

Recently, in plant-beneficial bacteria, bacterial secretion system has been hypothesized to play a role in suppressing plant pathogens (Tang et al., 2018).

In diseased fruits, the relative abundance of genes related to ribosome biogenesis in eukaryotes, RNA transport, polycyclic aromatic hydrocarbon degradation, spliceosome, tyrosine metabolism, amino sugar and nucleotide sugar metabolism, glycine, serine and threonine metabolism were higher compared with healthy fruits (Fig. 9). The high abundance of polycyclic aromatic hydrocarbon (PAH) degradation genes indicates that microbes are involved in biodegradation of pollutants (toxic compounds on fruit surface) to utilize PAH as carbon and energy source (Hamann et al., 1999). The presence of toxic compounds on diseased fruits could be a plausible explanation of decreased microbial diversity. We also observed that the genes related to amino sugar and nucleotide sugar metabolism were more abundant in diseased fruits. This phenomenon ratifies that the diseased fruit microbes are engaged in energy conversion and carbohydrate digestion (Yang et al., 2014). Another interesting finding was that the glycine, serine and threonine metabolism pathway associated genes were enriched in diseased fruits compared with healthy fruits. A recent study indicated that the glycine, serine and threonine metabolism is an imperative pathway contributing to bacterial sensitivity to antibiotics (Ye et al., 2018). It could be associated with decreased bacterial diversity in fruit microbiome. These results showed that the infection by postharvest pathogens not only shift the diversity and composition of fruit microbiome but also lead to the predicted microbiome functioning.

Conclusion: Our results indicated that food-borne pathogen infection disrupt the fruit microbiome and had lower microbial diversity in diseased fruit compared with healthy fruits. The structure and composition of fruit microbiome were significantly altered between healthy and diseased fruits resulting in the clear reduction of beneficial microorganism coupled with a high abundance of fruit pathogenic microbes in diseased fruits. Interestingly, we also detected previously unreported fruit pathogenic fungus Diplodia seriata on kiwifruit signifying the potential of metagenomic sequencing in deciphering previously unrecognized postharvest pathogens on specific hosts. Moreover, shotgun metagenomic sequencing provided meaningful insights about the functions of the microbiome in healthy and pathogen-infected fruits. However, further studies are still warranted to thoroughly understand the functional potential of fruit native microbiota and to construct synthetic microbial consortia for management of postharvest diseases.

Acknowledgments: This work was supported by grants from the Guizhou Science and Technology Fund Project (NO.: [2016]1006), the Natural Science Foundation of the Department of Education of Guizhou Province (NO.: qianjiao he KY word [2016]091), the Guiyang Science and Technology Bureau and Guiyang University Special Project (No.: GYU-KYZ[2018]01-05) and China Postdoctoral Science Foundation (NO.: 2017M623070). We thank Syed Adeel Zafar from Chinese Academy of Agricultural Sciences for his valuable suggestions for the organization of this paper.

\section{REFERENCES}

Abbas, M.F. and F. Naz. 2018. First report of Diplodiaseriata causing fruit rot of loquat in Pakistan. J. Plant Pathol. 100:325.

Abdelfattah, A., D. Ruano-Rosa, S.O. Cacciola, M.G.L.D. Nicosia and L. Schena. 2018. Impact of Bactroceraoleae on the fungal microbiota of ripe olive drupes. PloS One 13:e0199403.

Abdelfattah, A., M. Wisniewski, S. Droby and L. Schena. 2016. Spatial and compositional variation in the fungal communities of organic and conventionally grown apple fruit at the consumer point-of-purchase. Hortic. Res. 3:16047.

Bai, Y., D.B. Müller, G. Srinivas, R. Garrido-Oter, E. Potthoff, M. Rott, N. Dombrowski, P.C. Münch, S. Spaepen, M. Remus-Emsermann, B. Hüttel, A.C. McHardy, J.A. Vorholt and P. Schulze-Lefert. 2015. Functional overlap of the Arabidopsis leaf and root microbiota. Nature 528:364-369. 
Berendsen, R.L., C.M. Pieterse and P.A. Bakker. 2012. The rhizosphere microbiome and plant health. Trends Plant Sci. 17:478-486.

Bulgarelli, D., K. Schlaeppi, S. Spaepen, E.V.L. van Themaat and P. Schulze-Lefert. 2013. Structure and functions of the bacterial microbiota of plants. Ann. Rev. Plant Biol. 64:807-838.

Bulgarelli, D., M. Rott, K. Schlaeppi, E.V.L.V. Themaat, N. Ahmadinejad, F. Assenza, P. Rauf, B. Huettel, R. Reinhardt, E. Schmelzer, J. Peplies, F.O. Gloeckner, R. Amann, T. Eickhorst and P. Schulze-Lefert. 2012. Revealing structure and assembly cues for Arabidopsis root-inhabiting bacterial microbiota. Nature 488:91-95.

Bulgarelli, D., R. Garrido-Oter, P.C. Münch, A. Weiman, J. Dröge, Y. Pan, A.C. McHardy and P. Schulze-Lefert. 2015. Structure and function of the bacterial root microbiota in wild and domesticated barley. Cell Host Microbe 17:392-403.

Chou, M.Y., J.V. Heuvel, T.H. Bell, K. Panke-Buisse and J. Kao-Kniffin. 2018. Vineyard under-vine floor management alters soil microbial composition, while the fruit microbiome shows no corresponding shifts. Sci. Rep. 8:11039.

Cook, D.W.M., P.G. Long and S. Ganesh. 1999. The combined effect of delayed application of yeast biocontrol agents and fruit curing for the inhibition of the postharvest pathogen Botrytis cinerea in kiwifruit. Postharvest Biol. Technol. 16:233-243.

Di Francesco, A., M. Mari, L. Ugolini and E. Baraldi. 2018. Effect of Aureobasidium pullulans strains against Botrytis cinerea on kiwifruit during storage and on fruit nutritional composition. Food Microbiol. 72:67-72.

Diskin, S., O. Feygenberg, D. Maurer, S. Droby, D. Prusky and N. Alkan. 2017. Microbiome alterations are correlated with occurrence of postharvest stem-end rot in mango fruit. Phytobiomes 1:117-127.

Droby, S. and M. Wisniewski. 2018. The fruit microbiome: A new frontier for postharvest biocontrol and postharvest biology. Postharvest Biol. Technol. 140:107-112.

Droby, S., M. Wisniewski, D. Macarisin and C. Wilson. 2009. Twenty years of postharvest biocontrol research: is it time for a new paradigm? Postharvest Biol. Technol. 52:137-145.

Habiba, R. Noreen, S.A. Ali, K.A. Hasan, V. Sultana, J. Ara and S. Ehteshamul-Haque. 2019. Evaluation of biocontrol potential of epiphytic yeast against postharvest Penicillium digitatum rot of stored Kinnow fruit (Citrus reticulata) and their effect on its physiochemical properties. Postharvest Biol. Technol. 148:38-48.

Hamann, C., J. Hegemann and A. Hildebrandt. 1999. Detection of polycyclic aromatic hydrocarbon degradation genes in different soil bacteria by polymerase chain reaction and DNA hybridization. FEMS Microbiol. Lett. 173:255-263.

Hamid, M.I., M. Hussain, M.U. Ghazanfar, M. Raza and X.Z. Liu. 2014. Trichothecium roseum causes fruit rot of tomato, orange, and apple in Pakistan. Plant Dis. 98:1271.

Hamid, M.I., M. Hussain, Y. Wu, X. Zhang, M. Xiang and X. Liu. 2017. Successive soybean-monoculture cropping assembles rhizosphere microbial communities for the soil suppression of soybean cyst nematode. FEMS Microbiol. Ecol. 93:fiw222.

Hayat, F., K.M. Nawaz, S.A. Zafar, R.M. Balal, N.M. Azher, A.U. Malik and B.A. Saleem. 2017. Surface coating and modified atmosphere packaging enhances storage life and quality of 'Kaghzi lime'. J. Agr. Sci Tech. 19:11511160.

Hussain, M., M.I. Hamid, J. Tian, J. Hu, X. Zhang, J. Chen, M. Xiang and X. Liu. 2018. Bacterial community assemblages in the rhizosphere soil, root endosphere and cyst of soybean cyst nematode-suppressive soil challenged with nematodes. FEMS Microbiol. Ecol. 94:fiy142.

Hussain, M., M.I. Hamid, M.U. Ghazanfar, N. Akhtar and M. Raza. 2016. First report of fruit rot of strawberry caused by Geotrichum candidum in Pakistan. Plant Dis. 100:1948.

Kim, Y.K., J.H. Kwak, C.G. Aguilar and C.L. Xiao. 2016. First report of black rot on apple fruit caused by Diplodia seriata in Washington State. Plant Dis. 100:1499-1499.

Latocha, P., R. Wołosiak, E. Worobiej and T. Krupa. 2013. Clonal differences in antioxidant activity and bioactive constituents of hardy kiwifruit (Actinidia arguta) and its year-to-year variability. J. Sci. Food Agric. 93:14121419.

Lorenzini, M. and G. Zapparoli. 2018. Identification of Pestalotiopsis bicilita, Diplodia seriata and Diaporthe eres causing fruit rot in withered grapes in Italy. Eur. J. Plant Pathol. 151:1089-1093.

Mari, M., A. Spadoni and G. Ceredi. 2015. Alternative technologies to control postharvest diseases of kiwifruit. Stewart Postharvest Rev. 11:1-5.

Mendes, R., M. Kruijt, I. De Bruijn, E. Dekkers, M.V.D. Voort, J.H. Schneider, Y.M. Piceno, T.Z. DeSantis, G.L. Anderson, P.A.H.M. Bakker and J.M. Raaijmakers. 2011. Deciphering the rhizosphere microbiome for disease-suppressive bacteria. Sci. 332:1097-1100.

Moral, J., F. Luque and A. Trapero. 2008. First report of Diplodia seriata, the anamorph of "Botryosphaeria" obtusa, causing fruit rot of olive in Spain. Plant Dis. 92:311-311.

Mwaheb, M.A., M. Hussain, J. Tian, X. Zhang, M.I. Hamid, N.A. El-Kassim, G.M. Hassan, M. Xiang and X. Liu. 2017. Synergetic suppression of soybean cyst nematodes by chitosan and Hirsutella minnesotensis via the 
assembly of the soybean rhizosphere microbial communities. Biol. Control 115:85-94.

Panke-Buisse, K., A.C. Poole, J.K. Goodrich, R.E. Ley and J. Kao-Kniffin. 2015. Selection on soil microbiomes reveals reproducible impacts on plant function. ISME J. 9:980-989.

Polat, Z., Q.N. Awan, M. Hussain and D.S. Akgül. 2017. First report of Phytopythium vexans causing root and collar rot of kiwifruit in Turkey. Plant Dis. 101:1058.

Preto, G., F. Martins, J.A. Pereira and P. Baptista. 2017. Fungal community in olive fruits of cultivars with different susceptibilities to anthracnose and selection of isolates to be used as biocontrol agents. Biol. Control 110:1-9.

Ritpitakphong, U., L. Falquet, A. Vimoltust, A. Berger, J.P. Métraux and F. L'Haridon. 2016. The microbiome of the leaf surface of Arabidopsis protects against a fungal pathogen. New Phytol. 210:1033-1043.

Santhanam, R., A. Weinhold, J. Goldberg, Y. Oh and I.T. Baldwin. 2015. Native root-associated bacteria rescue a plant from a sudden-wilt disease that emerged during continuous cropping. Proc. Natl. Acad. Sci. USA 112:E5013-E5020.

Tang, J., Y. Liu, H. Li, L. Wang, K. Huang and Z. Chen. 2015. Combining an antagonistic yeast with harpin treatment to control postharvest decay of kiwifruit. Biol. Control 89:61-67.

Tang, J.Y., N.P. Bullen, S. Ahmad and J.C. Whitney. 2018. Diverse NADase effector families mediate interbacterial antagonism via the type VI secretion system. J. Biol. Chem. 293:1504-1514.

Tiwari, S., S.B. Jamal, S.S. Hassan, P.V. Carvalho, S. Almeida, D. Barh, P. Gosh, A. Silva, T.L.P. Castro and V. Azevedo. 2017. Two-component signal transduction systems of pathogenic bacteria as targets for antimicrobial therapy: an overview. Front. Microbiol. 8:1878.
Urbina, H., M.F. Breed, W. Zhao, K.L. Gurrala, S.G. Andersson, J. Ågren, S. Baldauf and A. Rosling. 2018. Specificity in Arabidopsis thaliana recruitment of root fungal communities from soil and rhizosphere. Fungal Biol. 122:231-240.

Wadhams, G.H. and J.P. Armitage. 2004. Making sense of it all: bacterial chemotaxis. Nat. Rev. Mol. Cell Biol. 5:1024-1037.

Wei, Z., J. Hu, Y.A. Gu, S. Yin, Y. Xu, A. Jousset, Q. Shen and V.P. Friman. 2018. Ralstonia solanacearum pathogen disrupts bacterial rhizosphere microbiome during an invasion. Soil Biol. Biochem. 118:8-17.

Wu, L., J. Wang, H. Wu, J. Chen, Z. Xiao, X. Qin, Z. Zhang and W. Lin. 2018. Comparative metagenomic analysis of rhizosphere microbial community composition and functional potentials under Rehmannia glutinosa consecutive monoculture. Int. J. Mol. Sci. 19:2394.

Yang, Y., K. Yu, Y. Xia, F.T. Lau, D.T. Tang, W.C. Fung, H.P. Fang and T. Zhang. 2014. Metagenomic analysis of sludge from full-scale anaerobic digesters operated in municipal wastewater treatment plants. Appl. Microbiol. Biotechnol. 98:5709-5718.

Ye, J.Z., X.M. Lin, Z.X. Cheng, Y.B. Su, W.X. Li, F.M. Ali, J. Zhang and B. Peng. 2018. Identification and efficacy of glycine, serine and threonine metabolism in potentiating kanamycin-mediated killing of Edwardsiella piscicida. J. Proteomics 183:34-44.

Zarraonaindia, I., S.M. Owens, P. Weisenhorn, K. West, J. Hampton-Marcell, S. Lax, N.A. Bokulich, D.A. Mills, G. Martin, S. Taghavi, D.V.D Lelie and J.A. Gilbert. 2015. The soil microbiome influences grapevine-associated microbiota. MBio 6:e02527-14.

Zhou, Y., G. Gong, Y. Cui, D. Zhang, X. Chang, R. Hu, N. Liu and $X$. Sun. 2015.Identification of Botryosphaeriaceae species causing kiwifruit rot in Sichuan Province, China. Plant Dis. 99:699-708. 